\title{
Platelet Aggregation Capacity of Clopidogrel Bisulphate Inhibited by Rabeprazole and Simvastatin in Patients Undergoing Myocar- dial Infarction in a Tertiary Care System: Drug Interaction Study
}

\author{
Jinesh Bahubali Nagavi ${ }^{*}$ and Bannimath Gurupadayya ${ }^{2}$ \\ ${ }^{1}$ Department of Pharmaceutical Chemistry, Sarada Vilas College of Pharmacy, India \\ ${ }^{2}$ Department of Pharmaceutical Analysis, JSS College of Pharmacy, JSS University, India
}

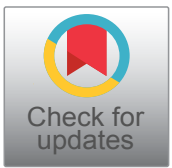

*Corresponding author: Jinesh Bahubali Nagavi, Department of Pharmaceutical Chemistry, Sarada Vilas College of Pharmacy, Mysore, Karnataka, 570004, India, Tel No: +919738516415

\begin{abstract}
Background: Proton pump inhibitors and statins reduce the effectiveness of clopidogrel in inhibiting the platelet aggregation. Clopidogrel, a prodrug adheres to CYP2C19, a hepatic enzyme to convert to its active metabolite in order to provide expected therapeutic action. Statins, mainly simvastatin metabolize through cytochrome P450 3A4, which also metabolizes clopidogrel partially. The combination of clopidogrel and PPI's are co-administered in patients going through ST segment elevated Myocardial infarction (STEMI), cardiac stent and percutaneous coronary intervention $(\mathrm{PCl})$.
\end{abstract}

Objectives of the study:

1. To determine the potential drug-drug interactions among patients on dual antiplatelets, PPIs and statins.

2. To determine the demographic and clinical characteristics of the patients to understand the competitive metabolism of the drugs.

3. To estimate the platelet aggregation effect of dual antiplatelets in presence of PPI's and statins.

Methodology: In the present study, potential drug-drug interactions ( $\mathrm{pDDI}$ ) was analyzed in the patients on dual antiplatelet therapy (DAPT) along with proton pump inhibitors and patients on dual antiplatelet therapy with proton pump inhibitors and statins. Platelet aggregation was measured in 116 patients undergoing ST segment elevated Myocardial infarction (STEMI), cardiac stent and percutaneous coronary intervention $(\mathrm{PCl})$ with clopidogrel bisulphate and aspirin along with PPI's and statins.

\begin{abstract}
Results: In the current study, Rabeprazole and Simvastatin, but not Omeprazole and rosuvastatin, decreased the antiplatelet activity of clopidogrel. The percent platelet aggregation was $81 \pm 5(p=0.001)$ and $33 \pm 10(p=0.027)$ in the presence of clopidogrel with Rabeprazole and pantoprazole respectively. Aggregation was found to be $91 \pm 4(p=0.001)$ and $22 \pm 03(p=0.031)$ in presence of clopidogrel with Simvastatin and rosuvastatin respectively.
\end{abstract}

Conclusion: A prominent drug-drug interaction was observed with patients on dual antiplatelet therapy along with Rabeprazole and Simvastatin.

\section{Keywords}

Clopidogrel, PPI's, Statins, STEMI, Antiplatelets, Platelet aggregation, Cytochrome P450, Potential drug-drug interaction

\section{Introduction}

Potential drug-drug interaction (pDDI) between dual antiplatelets (clopidogrel and aspirin) and clinically prescribed PPI's like Rabeprazole, pantoprazole, omeprazole [1] and statins such as Simvastatin, atorvastatin and rosuvastatin has been observed in many clinical studies. A very high mortality rate is associated with such interactions in a tertiary health care system. The competitive binding of several classes of drugs to a single metabolic enzyme cytochrome P450 which are most likely to be present in liver and other hepatic tissues, leading to drug-drug interaction. Due to competitive binding of two drugs to the single enzyme, the pharmacology of

Citation: Nagavi JB, Gurupadayya B (2020) Platelet Aggregation Capacity of Clopidogrel Bisulphate Inhibited by Rabeprazole and Simvastatin in Patients Undergoing Myocardial Infarction in a Tertiary Care System: Drug Interaction Study. Int J Clin Cardiol 7:188. doi.org/10.23937/2378-2951/1410188 Accepted: July 20, 2020; Published: July 22, 2020

Copyright: (C) 2020 Nagavi JB, et al. This is an open-access article distributed under the terms of the Creative Commons Attribution License, which permits unrestricted use, distribution, and reproduction in any medium, provided the original author and source are credited. 
both drugs alter [2], leading to major pharmacokinetic interaction.

In any health care system, drug-drug interactions have become a very important concern. The studies have significantly proven that that many drug-drug interactions can be explained by altering the metabolic enzymes that are present in the liver and other tissues. Almost all of the interactions between drugs are due to hepatic cytochrome P450 (P450 or CYP) enzymes being affected by previous administration of other drugs, which alter the pharmacokinetic behavior of a drug. In a multiple prescriptions, some of the drugs act as potent enzyme inducers, whereas others are inhibitors. Although, reports of enzyme inhibition are highly common than inducing. Therefore it becomes utmost important to understand the mechanisms of enzyme inhibition or induction in order to give appropriate multiple-drug therapies. Such studies will help to identify patients at greatest risk of drug interactions and adverse events to provide personalized medication [2].

Clopidogrel falls under the classification of thienopyridine drugs which decrease the platelet aggregation in patients to reduces coronary stent thrombosis and myocardial infarction and also those undergoing percutaneous coronary intervention $[3,4]$. Clopidogrel gets converted to its active metabolite by forming a disulfide bridge with adenosine diphosphate (ADP) receptor and exhibits antiplatelet effect [5-8]. In animals, especially rats it's found that cytochrome P450 $1 \mathrm{~A} 2$ is responsible for activation of clopidogrel [6], whereas in humans the activation of clopidogrel is mostly by the cytochrome P450 2C19 and partly by 3A4 [8].

Patients receiving antiplatelet therapy are most commonly prescribed with PPI's to reduce the gastrointestinal bleeding [9]. Cardiovascular events, stroke, myocardial infarction and mortality continue to occur in patients with vascular diseases because of competitive binding of the ADP receptor blocker clopidogrel and PPI's to the isoenzymes 2C19 and 3A4 [10,11]. Patients with acute coronary syndrome are most likely to have elevated levels of cholesterol which makes it necessary for statin therapy. Thus evaluation of each drug concentration in a poly prescription becomes at most important.

The American College of Cardiology/American Heart Association 2007 Unstable Angina/Non-ST-Segment Elevation Myocardial Infarction guidelines recommend concomitant PPI therapy with aspirin and clopidogrel in patients with a history of gastrointestinal bleeding [12]. Consequently, the number of patients affected by a PPI-clopidogrel interaction could be substantial. In fact, a combined total of 100 million prescriptions are written for both PPIs and clopidogrel annually [13]. However, this does not include all Rabeprazole use since, at some strengths, it is available over-the counter. It has been hypothesized that PPI use concurrently with clopi- dogrel will increase the risk of major adverse cardiac events [14].

Different isoforms of Cytochrome P450 (CYP) metabolized different types of substrates (or drugs molecule) and make them soluble during biotransformation. Therefore, fate of any drug molecule depends on how they are treated or metabolized by CYP isoform. There is a need to develop models for predicting substrate specificity of major isoforms of P450, in order to understand whether a given drug will be metabolized or not. In-silico method for predicting the metabolizing capability of major isoforms (e.g. CYP 3A4, 2D6, 1A2, 2C9 and 2C19) has been explained [15].

The main objective of the study is to determine the potential drug-drug interactions among patients on dual antiplatelets, PPIs and statins. Also demographic and clinical characteristics of the patients will help to understand the competitive metabolism of the drugs. Estimation of platelet aggregation among patients with $\mathrm{MI}$ and STEMI prescribed with dual antiplatelet therapy in presence of PPI's and statins.

Rabeprazole showed drastic decrease in the effect of clopidogrel tested by VASP phosphorylation. The clinical impact of the findings has a future scope of investigation, but treatment with PPIs at certain dose with the dual antiplatelet therapy is not recommended [16].

Studies also describes the use of Simvastatin a $\mathrm{CY}$ P3A4 substrate, competitively inhibits the activation of clopidogrel. Also explains about the use of statins not metabolized by CYP3A4 for a better treatment with clopidogrel [17].

\section{Methodology}

In this study, patients with myocardial infarction followed by stent implantation and other cardiovascular diseases and percutaneous coronary intervention, where prospectively assessed for platelet aggregation studies. Subjects prescribed with dual antiplatelet therapy having clopidogrel and aspirin along with PPI's and statins were investigated.

The institutional review board approved the protocol, and a written informed consent was signed by the patient/patient care taker, before commencing the study.

i. Study site: This study was conducted in JSS College of Pharmacy and Department of Cardiology, JSS Medical College and Hospital, Mysore.

ii. Study design: This was a prospective bioanalytical study.

iii. Study period: The study was conducted over a period of 15 months, from the month of October 2017 to December 2018.

iv. Study subjects: The study subjects were enrolled into the study based on the study criteria. 


\section{Study Criteria}

\section{Inclusion criteria}

1. Male or female between the ages of 40 to 60 years, inclusive who are admitted in the hospital (in-patients).

2. Females must have negative results for pregnancy tests performed: At Screening on a urine specimen obtained within 2 weeks prior to initial study drug administration.

3. Body Mass Index (BMI) is 19 to 26 , inclusive. BMI is calculated as weight in $\mathrm{kg}$ divided by the square of height measured in meters.

4. A condition of $\mathrm{MI}$, stroke, heart attack etc. with percutaneous coronary intervention admitted in the cardiology/other department in the hospital.

5. Patients receiving the above said medications.

6. Must voluntarily sign and date each informed consent, prior to the initiation of any screening or study-specific procedures.

\section{Exclusion criteria}

1. History of significant sensitivity to any drug.

2. Requirement for any over-the-counter and/or prescription medication other than above mentioned, vitamins and/or herbal supplements, on a regular basis.

3. Use of any medications (other than OTC/prescription), vitamins and/or herbal supplements, within the 1-week period prior to study drug administration.

4. Recent (6-month) history of drug or alcohol abuse.

5. Use of known inhibitors (e.g., ketoconazole) or inducers (e.g., carbamazepine) of cytochrome P450 3A (CYP3A) within 1 month prior to study drug administration.

\section{Investigation and study protocols}

The study protocols were approved by the institutional review board and written informed consent was obtained from the entire subjects before enrolling them to the study. In the study, 116 patients undergoing elective coronary artery stent implantation prescribed an oral loading dose of $600 \mathrm{mg}$ of clopidogrel (PLAVIXTM) followed by $75 \mathrm{mg} / \mathrm{d}$ for 28 days.

Participants signed up for the study had undergone a standard diagnostic procedure and treatment with primary percutaneous coronary intervention followed by stent implantation. All the patients were enrolled and studied prospectively. All the subjects received 300 $\mathrm{mg}$ of aspirin (NUSPRIN ${ }^{\mathrm{TM}}$ ) on admission and $200 \mathrm{mg} / \mathrm{d}$ thereafter throughout the study period.
The blood samples were withdrawn in the pathology laboratory $60 \pm 5$ minutes after administration of chewable aspirin (baseline) and the platelet aggregation capacity was tested.

Nineteen patients were on PPI's alone, among them twelve were prescribed with 20 mg of Rabeprazole (RABICIP-20 ${ }^{\mathrm{TM}}$ ) twice daily and seven were on Pantoprazole tablets IP (PANTOSEC ${ }^{\mathrm{TM}}$ ) $40 \mathrm{mg}$ once daily. Twenty five patients were on dual antiplatelet therapy along with these PPI's.

Out of twenty eight patients on statin therapy, seventeen were taking $40 \mathrm{mg}$ of Simvastatin (SIMVOTIN ${ }^{T M}$ ) a day, and eleven were taking $40 \mathrm{mg}$ ( $\mathrm{n}$ 5) of rosuvastatin (CRESTOR ${ }^{\mathrm{TM}}$ ) once daily. Platelet aggregation was measured before clopidogrel administration and 24 hours later at regular interval.

Platelet aggregation measurements were repeated in thirty one patients on clopidogrel and aspirin alone and thirty eight patients on dual antiplatelet therapy with PPI's and statins 24 days after successful stent implantation.

Based on the prescribed medications, the subjects were divided into 5 quartiles. Platelet aggregation was measured at $24 \mathrm{~h}$ and compared with the baseline at $0 \mathrm{~h}$ after the administration of clopidogrel as loading dose. Percentage aggregations were presented categorically in the 5 quartiles. To test the linear trend Mantel-Haenszel ${ }^{2}$ analysis was used. Patients of first quartile were compared with second till fifth quartiles using a 2-tailed fisher's test. Variables were presented as mean \pm SD.

Baseline demography and clinical characteristics of patients on dual antiplatelet therapy, PPI's and statins individually and in combinations are mentioned in Table 1.

\section{Evaluation of Platelet Aggregation}

\section{Preparation of Platelet rich plasma (PRP)}

Platelet-rich plasma was prepared by centrifugation of $5 \mathrm{~mol} / \mathrm{L}$ of ADP and was measured with a dual channel Aggregometer (model 440, Chronolog, Havertown, PA). Chart recordings were monitored on a Kipp-Zone Chart Recorder (Fisher Scientific, Chicago, IL).

The whole blood collected from the patients was anticoagulated with sodium citrate (sodium citrate/whole blood ratio, 1:10) and centrifuged at $10000 \mathrm{rpm}$ for 10 minutes at room temperature for preparation of platelet rich plasma (PRP). The prepared PRP was incubated for 30 mins at $37^{\circ} \mathrm{C}$ in capped tubes with ${ }^{14} \mathrm{C}$-serotonin. The cloudy yellow supernatant containing the platelets was separated without disturbing the WBC and RBC cell layers. Platelet-poor plasma (PPP) was prepared by centrifuging the remaining sample at $10000 \mathrm{rpm}$ for $20 \mathrm{~min}$ utes at room temperature [18]. Platelet function testing 


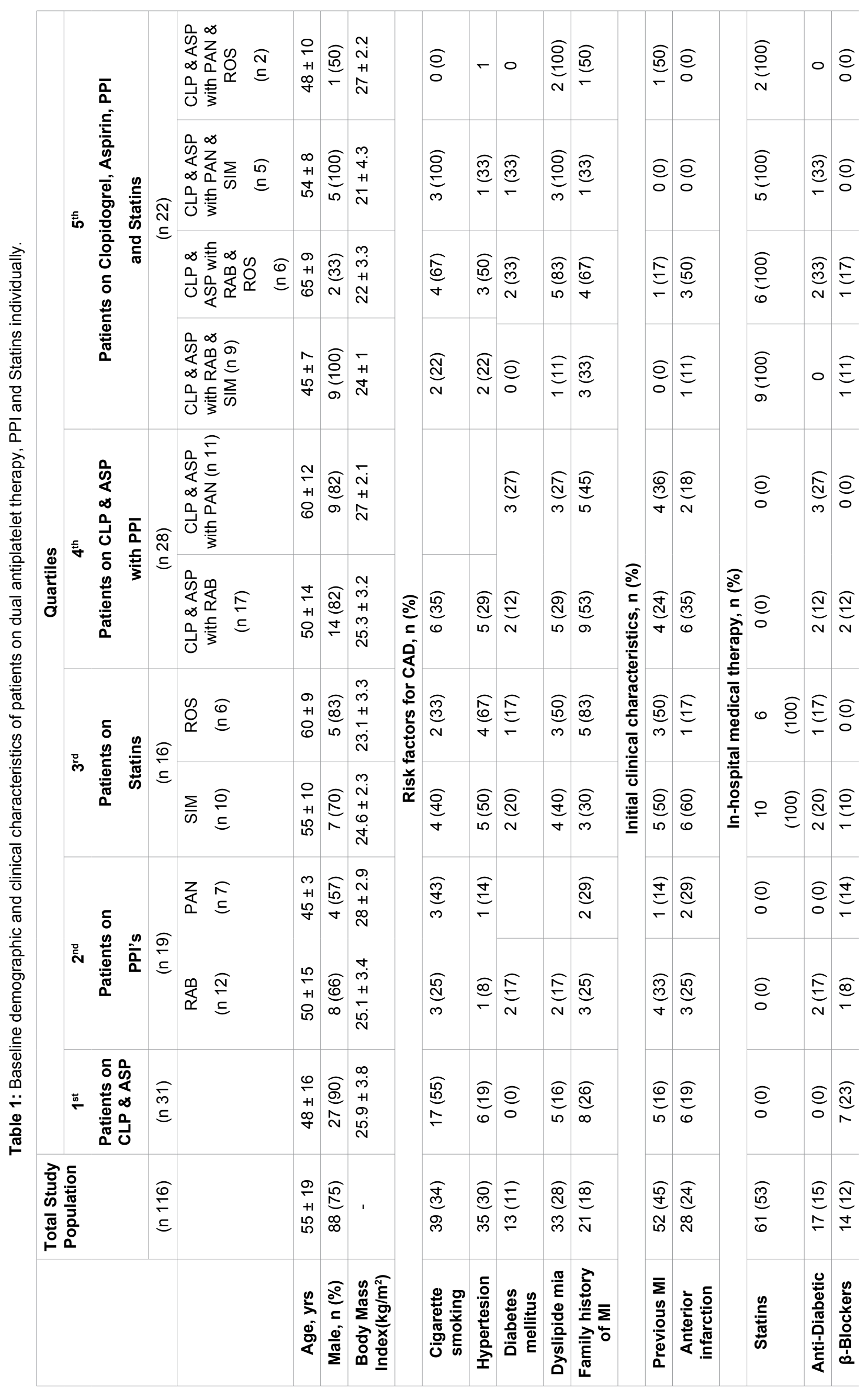


was completed within 3 hours after blood collection. The PRP was adjusted to a platelet count of 200 to 350 $\times 10^{3} / \mu \mathrm{L}\left(200-350 \times 10^{9} / \mathrm{L}\right)$ after addition of PPP. Clinical samples should be assayed with similar platelet counts in the normal range. Platelet counts less than $100 \times 10^{3} /$ $\mu \mathrm{L}\left(100 \times 10^{9} / \mathrm{L}\right)$ are not optimal for these functional tests.

\section{Evaluation}

Platelet aggregation was determined by measuring the change in the optical density (i.e., light transmittance) of stirred PRP after addition of the aggregating agent to the aggregometer cuvette. Platelet aggregation occurs only if the PRP in the aggregometer cuvette is stirred, usually at the rate of 800 to $1,200 \mathrm{rpm}$. A Teflon-coated magnetic stirrer was used. The aggregometer was standardized by placing the patient's PPP sample in one channel to represent $100 \%$ light transmittance and the patient's PRP sample in another channel representing $0 \%$ transmittance. The increase in light transmittance from $0 \%$ to $100 \%$ is reflected on the chart recorder as the aggregometer tracing. Usually, the baseline of the patient's PRP was adjusted to be at the $10 \%$ chart deflection level, and the patient's PPP baseline was adjusted to the $90 \%$ level on the chart recorder. The light transmittance of the PRP relative to the PPP blank was recorded automatically. When an aggregating agent is added to the PRP, platelet aggregates form, and this event results in an increase in the light transmittance, which is recorded and used as an index of platelet aggregation.

\section{Calculations of platelet aggregation}

The percentage of aggregation was determined as the percentage of chart deflection between $10 \%$ and $90 \%$ at its highest average point of deflection on the chart recorder as follows:

Percentage of Aggregation $=($ Highest Average Point of Deflection - 20)/80 × 100 .

\section{Statistical Analysis}

Platelet activity was expressed as a percentage of baseline value. Each patient served as his or her own control.
Statistical analysis was performed using the Graph pad Prism Software (version 5.00, Graph pad Software, Inc., San Diego, California). We estimated that a study sample size of 61 patients would enable a one-half standard deviation difference ( $10 \%$ difference in platelet aggregation between the groups) to be detected, with an $80 \%$ statistical power and a $5 \%$ alpha risk. Continuous variables are expressed as mean SD. Categorical variables are expressed as percentages. Comparisons between groups for the aggregation percentages were made with the chi-square or Fisher exact test for categorical variables and nonparametric statistical testing (Mann-Whitney) for continuous variables. Values of $p<$ 0.05 were considered statistically significant.

\section{Results}

Of the 116 patients enrolled, 88 (75\%) were male, mean age was $55 \pm 19$ years, and mean time from symptom onset to admission was $2.8 \pm 2$ hours.

\section{Evaluation of platelet aggregation}

Platelet aggregation studies showed a decrease in the percentage aggregation after $24 \mathrm{~h}$ in all the seven combination of patient plasma samples. The percentage aggregation is shown in Table 2.

\section{Effect of dual antiplatelet therapy}

In this study, the percentage platelet aggregation of plasma collected after $8 \pm 0.5$ hours of clopidogrel administration showed $35 \%$ inhibition compared to baseline of 0 hour (P- 0.0011 ) in the first section or quartile of participants. But, in the patients prescribed on dual antiplatelets also with PPI's and statins, platelet aggregation was reduced only to $78 \%$.

The results indicated that the efficiency of dual antiplatelet therapy was reduced measurably and drastically in the presence of rabeprazole and simvastatin when compared to pantoprazole and rosuvastatin.

\section{Platelet aggregation effect}

The participating patients were divided into 5 quartiles and the platelet aggregation was studied after 24 hours with that of 0 hour baseline activity of each patient at regular interval. Patients in the first quartile

Table 2: Platelet aggregation of patient plasma samples

\begin{tabular}{|c|c|c|c|c|}
\hline \multirow[t]{2}{*}{ SI. No. } & \multirow[t]{2}{*}{ Drug } & \multirow[t]{2}{*}{ No. of Patients } & \multicolumn{2}{|c|}{ Percentage Aggregation (\%) } \\
\hline & & & $\mathbf{O h}$ & $24 \mathrm{~h}$ \\
\hline 1. & CLP \& ASP & 31 & 96 & 35 \\
\hline 2. & CLP \& ASP with RAB & 16 & 92 & 71 \\
\hline 3. & CLP \& ASP with PAN & 9 & 94 & 63 \\
\hline 4. & CLP \& ASP with RAB \& ROS & 6 & 90 & 59 \\
\hline 5. & CLP \& ASP with RAB \& SIM & 9 & 96 & 78 \\
\hline 6. & CLP \& ASP with PAN \& SIM & 5 & 91 & 70 \\
\hline 7. & CLP \& ASP with PAN \& ROS & 2 & 92 & 56 \\
\hline
\end{tabular}

Note: Average \% aggregation in each group in \pm SD. 
were not resistant to clopidogrel (35 $\pm 6 \%$ of baseline platelet aggregation). Platelet aggregation from the second to fourth quartile had not reduced significantly
(Table 2 ), at $71 \pm 3 \%, 63 \pm 4 \%$, and $57 \pm 7 \%$ of the respective baselines $(P<0.005$ for all, (Figure 1a). The results clearly indicated the inhibition in platelet aggregation

A
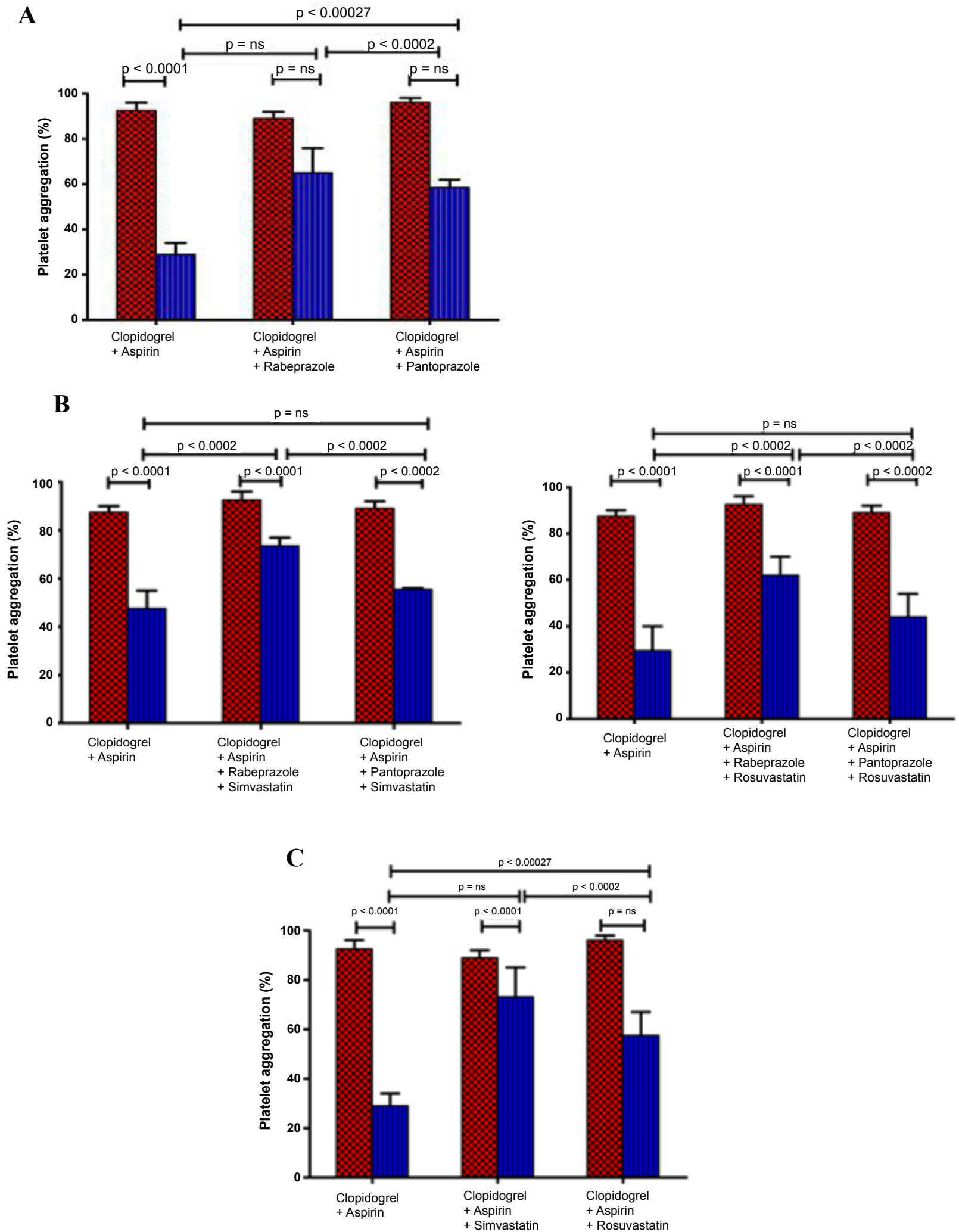

Figure 1: The in vivo effect of PPI's and statins on the dual antiplatelets activity of clopidogrel and aspirin. (A) Platelet aggregation at $0 \mathrm{~h}$ (dotted columns) and at $24 \mathrm{~h}$ (line columns) after clopidogrel and aspirin administration in patients treated without and with PPIs (40 mg); (B) The dual antiplatelets activity of clopidogrel and aspirin at $0 \mathrm{~h}$ (dotted columns) and at $24 \mathrm{~h}$ (line columns) in patients treated with and without PPI's and statins in different combinations; (C) Platelet aggregation at $0 \mathrm{~h}$ (dotted columns) and at $7 \mathrm{~h}$ (line columns) after clopidogrel and aspirin administration in patients with and without statins $(40 \mathrm{mg})$. 
decreased significantly in the first quartile ( $P$ for trend 0.01). Patients on dual antiplatelet therapy with Rabeprazole and Simvastatin showed no significant reduction in the platelet aggregation, $78 \pm 5 \%$ to baseline $96 \pm 4 \%$ $(p=n s)$. Platelet aggregation reduced considerably to $59 \pm 4 \%$ to the baseline $0 \mathrm{~h}(\mathrm{p}<0.005)$ in patients with dual antiplatelets, Rabeprazole and rosuvastatin.

\section{Clinical conclusion}

The baseline demographic and clinical characteristics of selected subjects had no significant change except the number of smokers decreased significantly and gradually from the first through fifth quartile (Table 1). It was also found that the other demographic parameters didn't differ, such as prescribed medications during hospital stay and in discharge summary (Table 1 ).

All the 116 patients in the investigation were monitored with a continuous clinical update. During the period six patients developed recurrent STEMI, three patients suffered from peripheral arterial occlusion, requiring immediate surgery. Two patients were reported with stent thrombosis followed by MI. Therefore, eleven patients had repeated cardiovascular events, seven of which occurred during treatment and four patients during the clinical follow-up. Among the eleven patients, five patients resistant to clopidogrel (fourth quartile) and six were from the fifth quartile. In variance, major bleeding occurred in one of the patient from fourth quartile.

Patients were senior citizens with repeated cardiovascular events ( $P$ 0.008), and had a lower percentage reduction of platelet aggregation which persisted up to 24 hours ( $90 \pm 16 \%$ vs. $72 \pm 7 \%$, P 0.001 , Figure 1 ).

\section{Discussion}

The comprehensive objective of cytochrome enzymes is to ease the removal of a lipophilic drug by converting it to hydrophilic in nature by drug metabolism [19]. The current study indicated that clopidogrel is less efficient in platelet aggregation inhibition when administered in combination with Rabeprazole, a CYP3A4 inhibitor and Simvastatin, a CYP2C19 substrate. In reverse, when clopidogrel was co administered with Rabeprazole and rosuvastatin, platelet aggregation inhibition was not altered due to a hydrophilic drug was not metabolized by the CYP system. Additionally, in vivo studies indicated that clopidogrel is converted to its active form predominantly by CYP2C19 isoenzyme and partially metabolized by CYP3A4, the most prominently expressed cytochrome isoenzyme in the human liver [20]. The authors based on the significant results conclude that Rabeprazole and Simvastatin inhibit CYP450 isoenzymes activity in a dose-dependent manner, at doses routinely administered to patients in cardiovascular diseases and therefore the metabolic conversion of clopidogrel to its pharmacologically active form decreased drastically.
Individualized attention and care to the patients is very much necessary because of polypharmacy, in which many drugs compete to get metabolized by $\mathrm{CY}$ P3A4, therefore it becomes highly important to understand the capacity and efficacy of clopidogrel, especially in presence of other medications [21]. Patients suffering from myocardial infarction, ST segment elevated MI, acute coronary syndromes, and those on percutaneous coronary intervention; clopidogrel is prescribed for as long as 4 months. Therefore, estimating the potential drug-drug interaction of clopidogrel with other drugs becomes extremely important.

Clopidogrel gets hydrolyzed from its methyl ester to inactive carboxylic acid derivative, after an oral dose of $75 \mathrm{mg}$. The inactive form of clopidogrel represents almost $85 \%$ of component related to clopidogrel in plasma. So only remaining around $15 \%$ of the active drug moiety is available for metabolism to its active form. Therefore the level of active clopidogrel is less than 1015 folds as that of inactive carboxylic acid form. Platelet function testing is also absolutely necessary to identify the aspirin resistant patients.

The lipophilic nature of simvastatin competitively inhibits the clopidogrel metabolism through CYP3A4. Simvastatin has been responsible for $75 \%$ of HMG-CoA reductase activity [22]. Simvastatin lactone binds majorly to CYP3A4 than any other substrate and causes drugdrug interaction.

Clopidogrel mainly depends upon CYP2C19 to get converted to its active moiety, but partially binds to $\mathrm{CY}$ P3A4 for metabolism [23]. Also the rate of competitive inhibition depends on the concentration and relative affinity between two substrates to the binding site of CYP2C19 and CYP3A4 [24]. If present in equivalent concentration, Rabeprazole and Simvastatin are potent inhibitors of most of the substrates. Rabeprazole inhibits CYP3A4 whereas Simvastatin inhibits CYP2C19, therefore inhibiting the antiplatelet action of clopidogrel.

Simvastatin binds CYP2C19 more tightly than any other substrates which are found in lower concentration. Simvastatin inhibits the metabolism of clopidogrel because of a very low concentration of clopidogrel that binds to CYP2C19 than Simvastatin itself.

An instrument which could measure the activated platelets by thrombin activating peptide to agglomerate fibrinogen beads [18], which provides fast, automated and reproducible results with small sample size, was used in this study [10]. A device which allows to measure platelet aggregation in which ADP is used to enhance platelet aggregation was required, because clopidogrel is itself an ADP receptor antagonist. The devices like turbidimetric aggregometer give indirect results and are dependent on analyst differences and also due to citrated platelet rich plasma containing various blood components. 
Literatures from decades describe about the fluctuating clopidogrel activity with the metabolism of cytochrome P450 isoenzyme, mostly 2C19 and partly $3 \mathrm{~A} 4$ are responsible to convert clopidogrel prodrug to its active form. Many other factors like receptor signal pathway and ADP receptor polymorphism also varies the platelet aggregation response by clopidogrel. Also it is been reported that cytochrome $\mathrm{P} 450$ isoenzyme, $\mathrm{CY}$ P3A4 is triggered mainly by polycyclic aromatic hydrocarbons which are present in cigarette smoke.

According to a randomized crossover study a metabolic drug interaction was found between clopidogrel and other PPIs except with Rabeprazole [25].

The major findings in the current study having a decent sample size comparatively, provides a confirmatory proof to conclude that the clopidogrel resistance is mainly because of the competitive binding to the enzyme CYP2C19 and CYP3A4. Clinically it could be very fatal to the patients on dual antiplatelet therapy with rabeprazole and simvastatin. By increasing the loading dose of clopidogrel may significantly reduce the percentage of recurrent cardiovascular cases. However resistance to clopidogrel was observed in MI patients.

\section{Conclusion}

Rabeprazole and simvastatin showed more clopidogrel resistance after eliminating the effects of inter-individual variability in clopidogrel metabolism, when compared to other proton pump inhibitors and HMG-CoA reductase enhancer treatment.

\section{Conflict of Interest}

There is no conflict of interest among the authors.

\section{Acknowledgement}

The authors express their sincere thanks to the Head of the Department, Dept. of Pharmaceutical Chemistry and The Principal, Sarada Vilas College of Pharmacy and JSS College of Pharmacy, JSS Academy of Higher Education and Research, Mysuru for providing the necessary facilities to carry out the research work.

Authors thank the physicians and the staff of JSS Hospital, Mysuru, Karnataka, India for their support towards the collection of sample from the patients and in analyzing the samples.

\section{References}

1. Carvalho RF, Albuquerque A (2010) Interacção clopidogrel - inibidores da bomba de protões: Implicações para a prática clínica. Rev Port Cardiol 29: 1555-1567.

2. Bibi Z (2008) Role of cytochrome $P 450$ in drug interactions. Nutr Metab 27: 1743-1752.

3. Bertrand ME, Rupprecht HJ, Urban P, Gershlick AH (2000) Double-blind study of the safety of clopidogrel with and without a loading dose in combination with aspirin compared with ticlopidine in combination with aspirin after coronary stenting: The Clopidogrel Aspirin Stent International Cooperative Study (CLASSICS). Circulation 102: 624-629.
4. Savi P, Herbert JM, Pflieger AM, Dol F, Delebassee D, et al. (1992) Importance of hepatic metabolism in the antiaggregating activity of the thienopyridine clopidogrel. Biochem Pharmacol 44: 527-532.

5. Savi P, Combalbert J, Gaich C, Rouchon MC, Maffrand JP, et al. (1994) The antiaggregating activity of clopidogrel is due to a metabolic activation by the hepatic cytochrome P450-1A. Thromb Haemost 72: 313-317.

6. Savi P, Pereillo JM, Uzabiaga MF, Combalbert J, Picard C, et al. (2000) Identification and biological activity of the active metabolite of clopidogrel. Thromb Haemost 84: 891896.

7. Geiger J, Brich J, Honig-Liedl P, Eigenthaler M, Schanzenbächer $P$, et al. (1999) Specific impairment of human platelet P2Y(AC) ADP receptor-mediated signaling by the antiplatelet drug clopidogrel. Arterioscler Thromb Vasc Biol 19: 2007-2011.

8. Sugidachi A, Asai F, Yoneda K, Iwamura R, Ogawa T, et al. (2001) Antiplatelet action of R-99224, an active metabolite of a novel thienopyridine-Type G(i)-linked P2T antagonist, CS-747. Br J Pharmacol 132: 47-54.

9. Bjornsson TD, Callaghan JT, Einolf HJ, Fischer V, Gan L, et al. (2003) The conduct of in vitro and in vivo drug-drug interaction studies: A Pharmaceutical Research And Manufacturers Of America (PhRMA) Perspective. Drug Metab Dispos 31: 815-832.

10. Quinn MJ, Fitzgerald DJ (1999) Ticlopidine and clopidogrel. Circulation 100: 1667-1672.

11. CAPRIE Steering Committee (1996) A randomized, blinded, trial of clopidogrel versus aspirin in patients at risk of ischaemic events (CAPRIE). Lancet 348: 1329-1339.

12. Norgard NB, Abu-Fadel M (2009) Comparison of prasugrel and clopidogrel in patients with acute coronary syndrome undergoing percutaneous coronary intervention. Vasc Health Risk Manag 5: 873-882.

13. Mehta A, Mehta D, Loganathan J, Paladugu N, Bhalodkar NC (2011) Clopidogrel with proton pump inhibitors: Safe or Not? Clin Cardiol 34: 528-531.

14. Chan FKL (2012) Anti-platelet therapy and managing ulcer risk. J Gastroenterol Hepatol 27: 195-199.

15. Mishra NK, Agarwal S, Raghava GPS (2010) Prediction of cytochrome P450 isoform responsible for metabolizing a drug molecule. BMC Pharmacol 10: 1471-1480.

16. Gilard Martine, Arnaud Bertrand, Cornily Jean-Christophe, Gal Grégoire Le, Lacut Karine, et al. (2008) Influence of Omeprazole on the antiplatelet action of clopidogrel associated with aspirin: The Randomized, Double-Blind OCLA (Omeprazole Clopidogrel Aspirin) Study. J Am Coll Cardiol 51: 256-260.

17. Lau WC, Gurbel PA, Watkins PB, Neer CJ, Hopp AS, et al. (2004) Contribution of hepatic cyto-chrome P450 3A4 metabolic activity to the phenomenon of clopidogrel resistance. Circulation 109: 166 -171.

18. Smith JW, Steinhubl SR, Lincoff AM, Coleman JC, Lee TT, et al. (1999) Rapid platelet-function assay: An automated and quantitative cartridge-based method. Circulation 99: 620-625.

19. Carville DG, Schleckser PA, Guyer KE, Corsello M, Walsh MM (1998) Whole blood platelet function assay on the ICHOR point of care hematology analyzer. J Extracorpor Technol 30: 171-177.

20. Lakkis NM, George S, Thomas E, Ali M, Guyer K, et al. 
(2001) Use of ICHOR-platelet works to assess platelet function in patients treated with GP IIb/llla inhibitors. Catheter Cardiovasc Interv 53: 346-351.

21. Tcheng JE, Talley JD, O'Shea JC, Gilchrist IC, Kleiman NS, et al. (2001) Clinical pharmacology of higher dose eptifibatide in percutaneous coronary intervention (The PRIDE Study). Am J Cardiol 88: 1097-1102.

22. Kearney AS, Crawford LF, Mehta SC, Radebaugh GW (1993) The interconversion kinetics, equilibrium, and solubilities of the lactone and hydroxyacid forms of the HMGCoA reductase inhibitor, Cl-981. Pharm Res 10: 1461-1465.
23. Lea AP, McTavish D (1997) Atorvastatin: A review of its pharmacology and therapeutic potential in the management of hyperlipidaemias. Drugs 53: 828-847.

24. Thummel KE, Wilkinson GR (1998) In vitro and in vivo drug interactions involving human CYP3A. Annu Rev Pharmacol Toxicol 38: 389-430.

25. Ferreiro JL, Angiolillo DJ (2011) Diabetes and antiplatelet therapy in acute coronary syndrome. Circulation 123: 798813. 